\title{
Using Hebbian Network for Cipher
}

\author{
Amera Istiqlal Badran \\ amera_istiqlal@uomosul.edu.iq \\ Nidal Hussein Al-Asadi \\ College of Computer Sciences and Mathematics \\ University of Mosul, Iraq
}

\section{Received on: 06/10/2008}

Accepted on: 04/12/2008

\section{ABSTRACT}

This research contains two parts, in the first part, a ciphering system is built using the classical Hebbian network to protect data against many expected threats during the transfer of the data. In the second part, deciphering has been built by using the Hebbian neural network.

The time has been calculate for both cipher and decipher. In the ciphering process, a Hebbian network has been developed through a qualitative primary weight which has large value. Then, an equation has been applied to minimize the weight matrix. Here, The idea of Stream Ciphering has been used so as to feed the network entries at the ciphering stage. The work has been applied by using (Visual Basic) language, issue (6.0) with the Object Oriented Programming (OOP) on a computer of the (P III, 600MHz) type.

Keywords: ciphering system, Hebbian network, Object Oriented Programming (OOP), Visual Basic) language.

$$
\begin{aligned}
& \text { استخدام شبكة (Hebbian) في التشفير }
\end{aligned}
$$

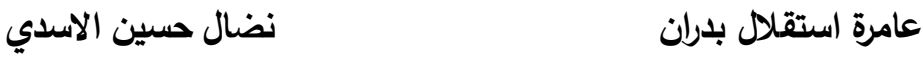

$$
\begin{aligned}
& \text { كلية علوم الحاسبات والرياضيات/ جامعة الدوصل بلدان }
\end{aligned}
$$

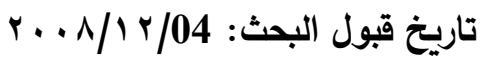

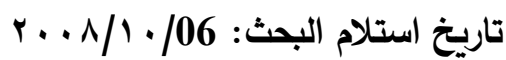

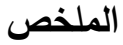

يحوي هذا البحث على جزئين:

في الجزء الأول تم بناء خوارزمية للتثفير عن طريق استخدام شبكة ( Hebbian ) التقليدية لحماية

البيانات ضد الكثير من التهديدات المتوقعة التي تتعرض لها أثناء نقل البيانات.أما الجزء الثاني فقد تم بناء خوارزمية لفك الثفرة عن طريق استخدام الثبكة العصبية(Hebbian).

وفي كلا الجزئين تم حساب الوقت المستغرق أي التثفير وفلك الثغرة لمعرفة كم من الوقت تستغرق. وفي

عملية التشفير تم تطوير شبكة ( Hebbian ) وذلك من خلال وزن أولي نوعي الذي يكون حجمه كبير، إذ تم

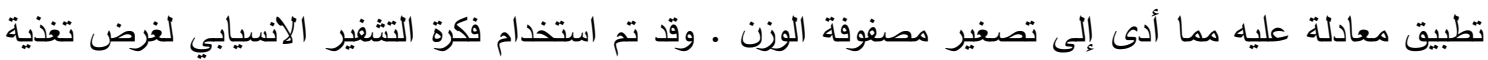
مداخل الثبكة في مرحلة التثفير • وضع هذا العمل قيد التطبيق باستخدام لغة (Visual Basic) الإصدار (6.0) مع أسلوب البرمجة الثيئية وطبق العمل على حاسبة من نوع (PIII,600MHz). الكلمات المفتاحية: نظام تشفير، شبكة Hebbian، أسلوب البرمجة الثيئية، لغة (Visual Basic).
} 
من الضروري التوجه إلى الثبكات العصبية الاصطناعية التي تعد من التطبيقات الحديثة في مجال

الذكاء الاصطناعي إذ اعتمدت على أسس بيولوجية في محاولة محاكاة السلوك البشري] [4] ل .

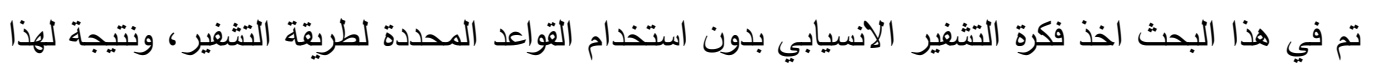

العمل تم تغذية شبكة (Hebbian) بإدخالات الملف وتم إجراء عملية التشفير باستخدام هذه الثبكة ومن ثم تمت لت

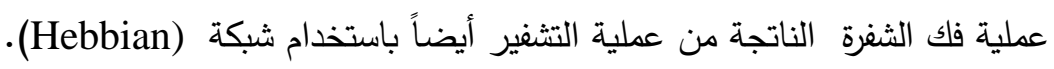

\section{:Hebbian شبكة}

لقد تم اكتثاف شبكة (Hebbian) من قبل العالم دونالد هيب (Donald Hebb)عام 1949. حيث قدم

العالم (Hebb) أول قاعدة لتعليم الثبكة العصبية اطلق عليها (Hebbian learning Rule) اعتمدت كقاعدة أساسية لتطوير خوارزميات التعليم[1][7]. وان الهدف من هذه الثبكة هو إعادة تعديل مصفوفة الوزن التي تمثل مصفوفة الارتباط بين العقد. أي انه في حالة تدريب شبكة (Hebbian) فان الأوزان بين عقد الثبكة سيتم تعديلها وفقاً لعلاقات التمثيل بين العقد. وقد تم اعتماد شبكة) (Hebbian) بصورة أساسية في إعادة تعديل مصفوفة الوزن فئن

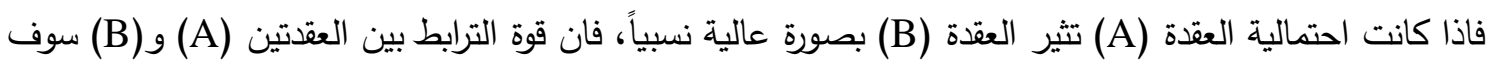
تزداد، وتسمى هذه الحالة بالتعلم من الذاكرة (Aearning from Memory) وذلك لان لان الثبكة سوف تستخدم

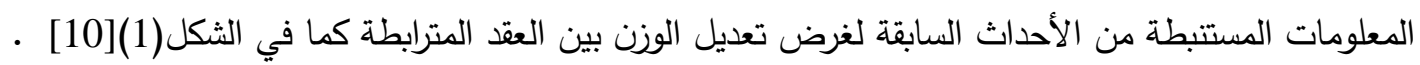

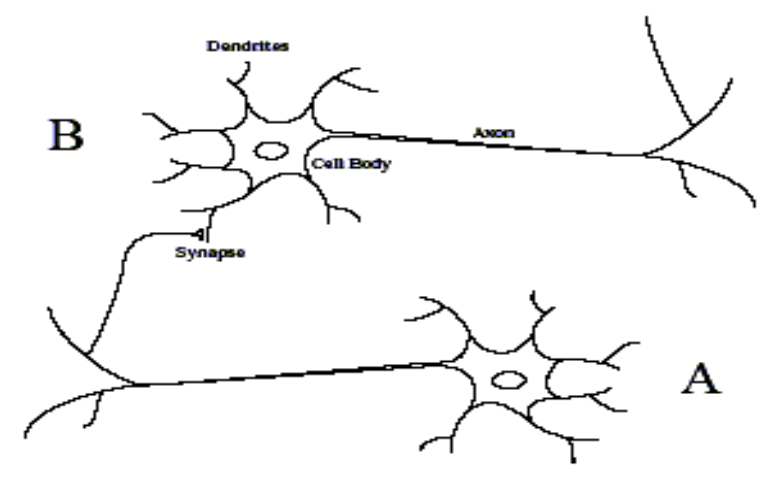

الثكل (1):يوضح تأثير عقدة على عقدة أخرى

إن نموذج التعلم لشبكة ( Hebbian ) يمكن أن يقع تحت أسلوبين وهما: التدريب بمعلم، والتدريب بدون معلم [9]. وقد تم استخدام شبكة ( Hebbian ) بدون معلم في هذا البحث، وتمتاز شبكة (Hebbian)من نوع بدون معلم بأنها تتكون من طبقة واحدة، أما اتجاه سير العمل فيها يكون من نوع السير إلى الأمام. وتمتاز هذه الثبكة

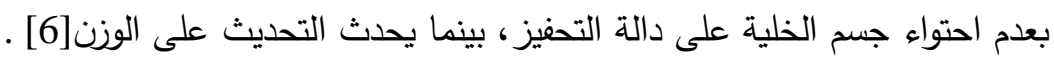


معمارية شبكة ( Hebbian ): يبين الثكل(2) الآتي بصورة عامة معمارية شبكة ( Hebbian ) التي تتكون من طبقة واحدة ويتم فيه توضيح الإدخالات والاخراجات.

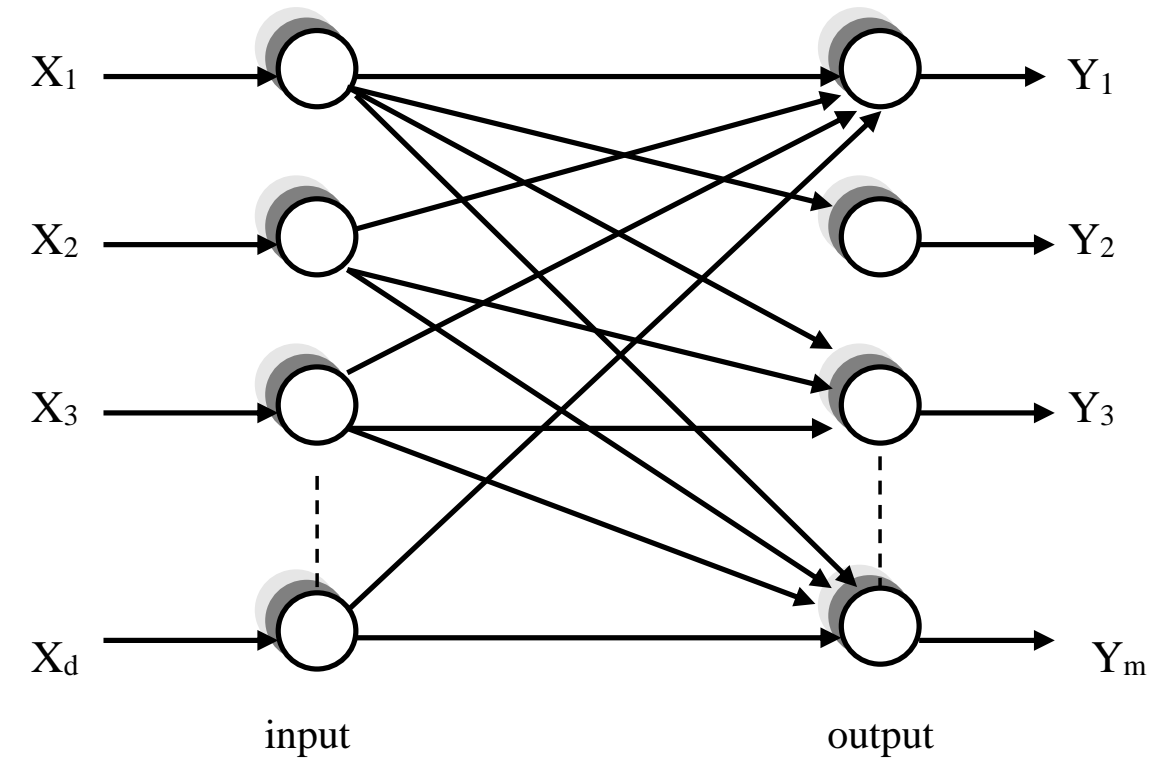

الثكل(2):مخطط يوضح معمارية شبكة (Hebbian)

لأجل الحصول على قيم الاخراج يمكن متابعة المعادلة الآتية:-

$y_{j}(n)=\sum_{i=1}^{d} W_{j i}(n) * X_{i}(n) \rightarrow j=1,2, \ldots \ldots, m$

• • ل الأجل تعديل مصفوفة الوزن فيمكن متابعة المعادلة الآتية:-

$\Delta W_{I J}(n)=\eta(n) y_{j}(n)\left[X_{i}(n)-\sum_{k=1}^{j} W_{k i}(n) y_{k}(n)\right]$

for $\quad i=1,2, \ldots \ldots . d \quad$ and $\quad j=1,2, \ldots \ldots . m$

$$
\begin{aligned}
& \text { حيث ان:- } \\
& \text {. تمثل قيمة الادخال i }
\end{aligned}
$$

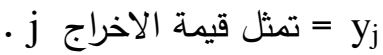

$$
\begin{aligned}
& \text {. تمثل قيمة مصفوفة الوزن = }
\end{aligned}
$$

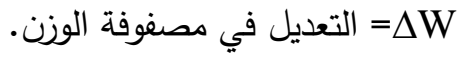

$$
\begin{aligned}
& \frac{1}{2}<\alpha \leq 1 \quad \frac{1}{n^{\alpha}}=1 \text { معامل التعلم }=\eta \\
& \text { = عد قيم الاخراج. m } \\
& \text { d عدد قيم الادخال. d } \\
& \text { = ع عد الدورات. }
\end{aligned}
$$


خوارزمية شبكة Hebbian:-

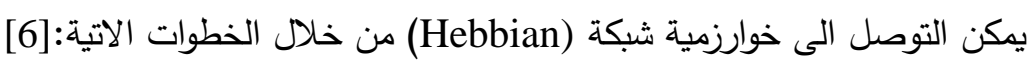

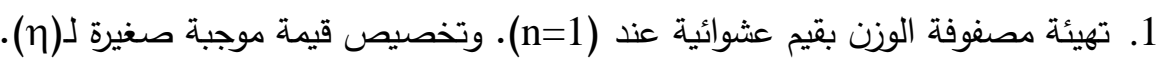

2. 2. حساب المعادلتان (1)ثم (2).

3. زيادة ( n ) بقيمة واحدة والذهاب إلى الخطوة الثانية، الاستمرار بالدوران إلى حد الوصول إلى القيمة الثابتة.

\section{[3]: لقد تم توضيح خطوات العمل في خوارزميتين الاتيتين}

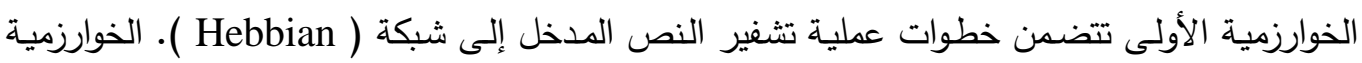
الثانية تتضمن خطوات فك تثفير النص باستخدام شبكة ( Hebbian ).وبعد تطبيق خوارزمية التشفير وفك الثفرة يتم حساب الوقت المستغرق لكل حالةً.ولقد تم استخدام شبكة (Hebbian ) في التشفير وذلك لان هذئ هذه الثبكة تمتاز بأنها خطية أي ان لها أسلوب خطي في عملية حساب الاخراجات[10], علماً ان هناك فوائد اخرى اضافية في التشفير اللاخطي ولكن تم استخدام هذه الطريقة لانها لا تحتوي على طبقة خفية مما يؤدي إلى زيادة سرعة

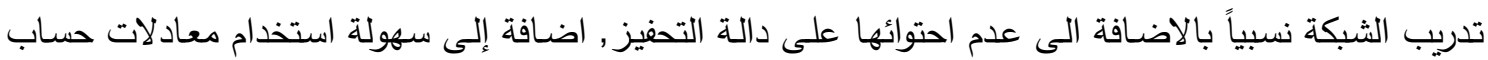
الاخراجات ومعادلات تعديل الوزن[6].

3. - 3وارزمية التشفير: • تبدأ عملية التثفير باستخدام شبكة (Hebbian) باعتماد الخوارزمية الآتية (الثكل(3))][3]:-

Input file

(ASCII code)

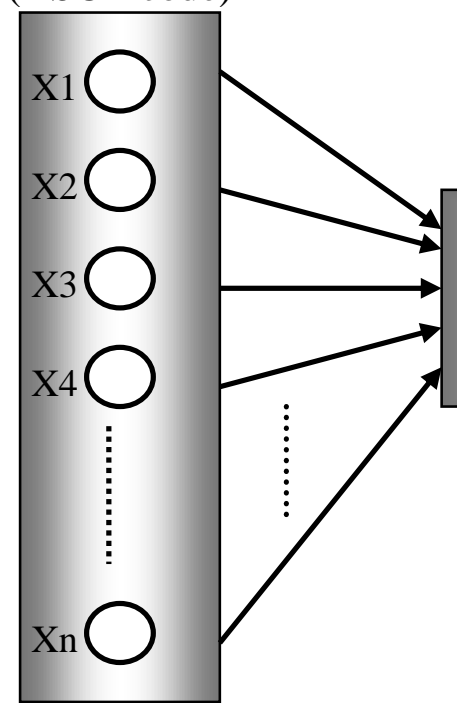

Output file (Flouting number)

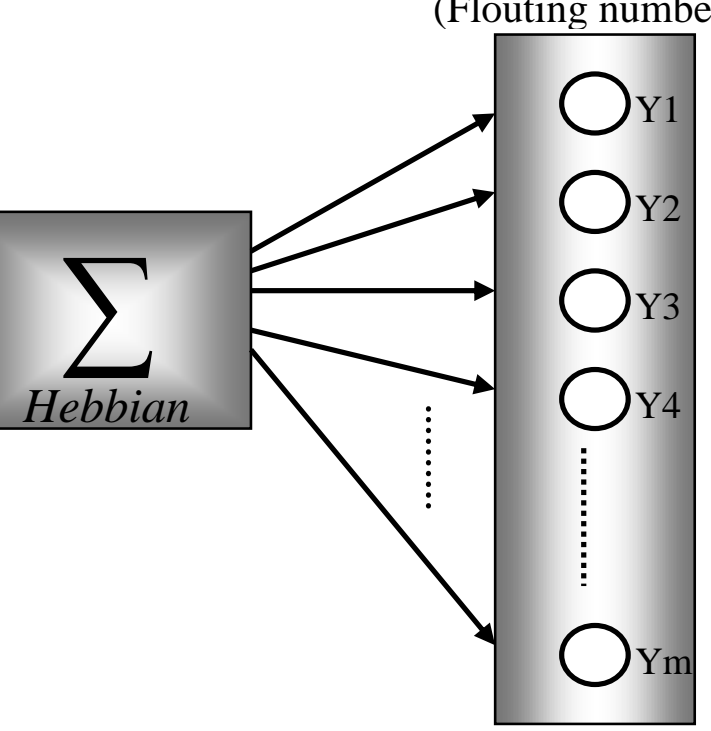

الثكل(3):مخطط عام لعملية التشفير باستخدام شبكة( Hebbian ) 


$$
\begin{aligned}
& \text { 1- فتح الملف المراد تثفيره . }
\end{aligned}
$$

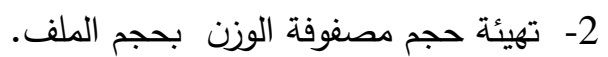

$$
\begin{aligned}
& \text { 3- توليد مفاتيح عشوائية وإحلالها إلى مصفوفة الوزن (الثكل (4)). }
\end{aligned}
$$

Input file

(ASCII code)

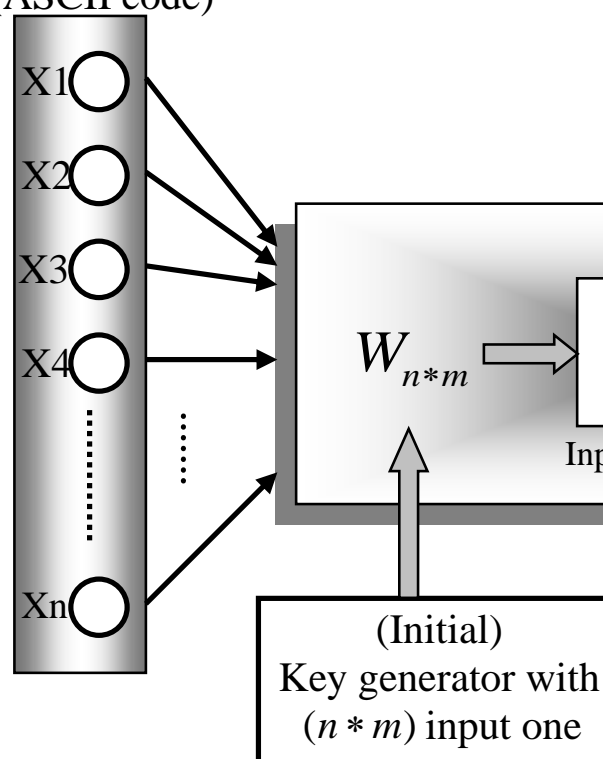

Output file

(Flouting number)

الثكل (4):مخطط تفصيلي لعملية التثفير باستخدام شبكة(Hebbian)

4- أجراء عملية تسوية(Normalization) لمصفوفة الوزن لتجنب حصول حالة تجاوز للقيم وذلك بالاعتماد على معادلة التبسيط الآتية:

$$
W_{i j}=W_{i j} / \sum W
$$

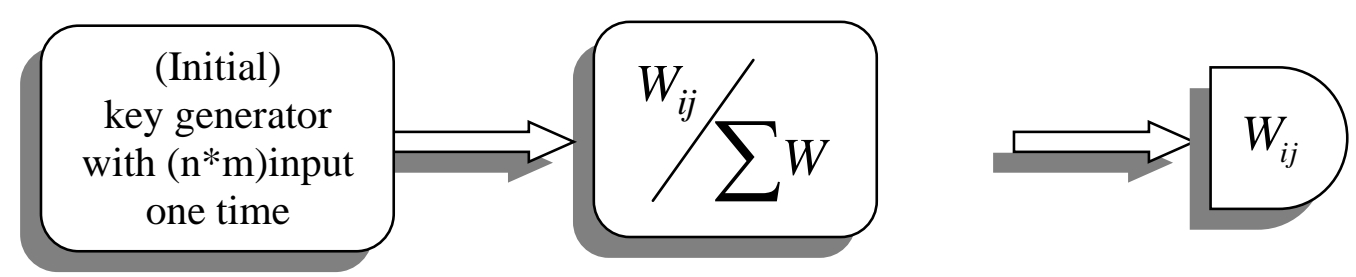

الثكل(5):مخطط تفصيلي يوضح عملية تسوية(Normalization) لمصفوفة الوزن 
إن إجراء عملية التعديل على مصفوفة الوزن وذلك من اجل تصغير قيم مصفوفة الوزن التي تم تكوينها

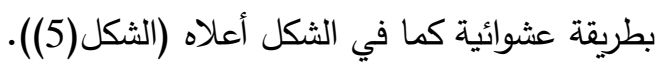

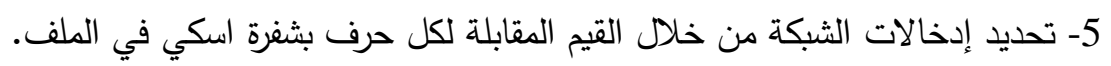

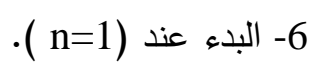

$$
\text { 7- تهيئة قيمة صغيرة لـ( ) ( }
$$

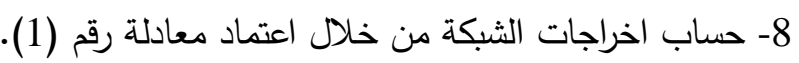

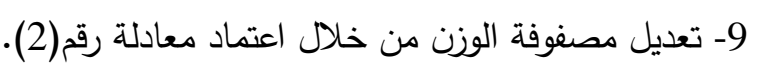

$$
\text { 10- 10 زيادة قيمة (n) بقيمة واحدة. }
$$

بعد الانتهاء من عملية التدريب لـ. n (n) لخطوات عدة، يتم الحصول على قيم الاخراجات التي تمثل القيم

المشفرة المقابلة لكل حرف مدخل. وتمثل عدد عقد الاخراجات بعدد عقد الإدخالات أي ان (d=m).

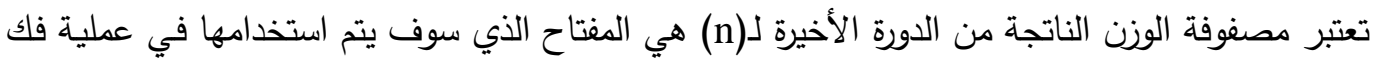

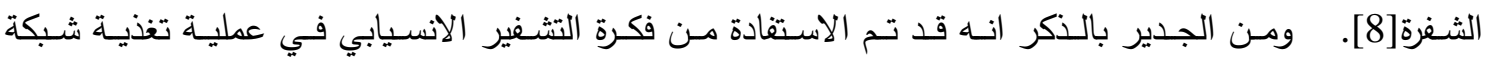
(Hebbian)

\section{4. خوارزمية فك الثفرة:}

تبدأ عملية فك الثفرة باستخدام شبكة ( Hebbian ) من خلال اعتماد الخوارزمية الآتية (الثكل(6))ر[3]:1- - فتح الملف المشفر وتهيئته كإدخالات . 2- تهيئة مصفوفة الوزن التي تمثل مصفوفة المفاتيح بقيم مصفوفة الوزن الناتجة من عملية التثفير . 3- حساب معكوس المصفوفة لمصفوفة الوزن، (أي اخذ آخر مصفوفة تم الحصول عليها من عملية التحديث على الوزن التي تم إجراءها بتطبيق معادلة رقم(2) ومن ثم حساب معكوس المصفوفة. 4- حساب اخراجات الثبكة من خلال اعتماد معادلة رقم (1). 5- تمثلت اخراجات الثبكة القيم الصريحة للنص الأصلي بشفرة اسكي. 


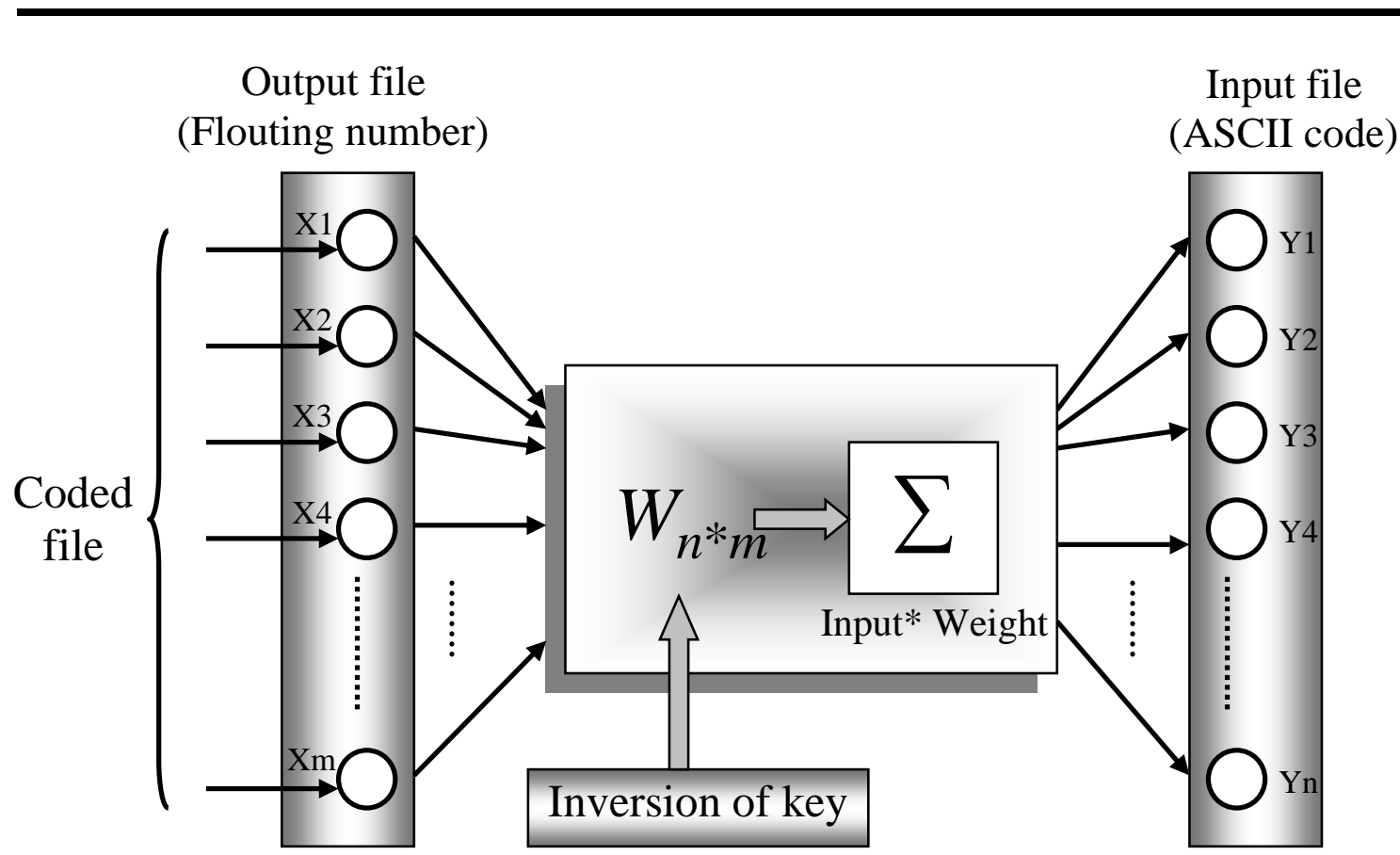

(6) الثكل)

مخطط تفصيلي لعملية فك الشفرة باستخدام شبكة(Debbian)

ولقد تم محاولة تهجين شبكة (Hebbian) مع الخوارزمية الجينية لعملية التشفير وفك الثفرة، إذ كانت

الخوارزمية الناتجة تسمى بالخوارزمية الهجينـة ولكن عند إجراء العمل تبين أن العملية تكاد أن تكون من الناحية فئه

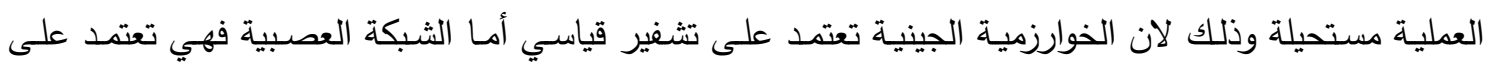
التشفير غير القياسي وبهذا أصبح وجود تتاقض ما بين الطريقتين بسبب هذا الاختلاف. وذلك لصعوبة تحديد معادلة مدى اللياقه[3].

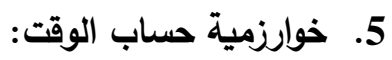
إن عملية حساب الوقت المستغرق في عملية التشفير أو فك الثفرة يتم حسابه باعتماد الخوارزمية الآتية:[3]

1- قراءة قيمة الوقت قبل عملية التشفير أو فك الثفرة بـ(دقيقة/ثانية). 2- إجراء عملية التثفير أو فك الثفرة. 3- قراءة قيمة الوقت بعد الانتهاء من عملية التشفير أو فلك الثفرة بـ(دقيقة/ثانية). 4- حساب الوقت لإجراء عملية التثفير أو فاك الثفرة(الوقت المستغرق) باعتماد المعادلة الآتية: الوقت المستغرق=الوقت بعد العملية(تشفير أو فك الثفرة)- الوقت قبل العملية(تشفير أو فك الثفرة). ان سبب استخدام خوارزمية لحساب الوقت لغرض التأكد من ان الوقت المستغرق لا يزيد عن الحد المعقول ولذلك تم قياس الوقت المستغرق وفيما بعد الثكل(8) يوضح قياس الوقت المستغرق للتشفير وفك الثفرة وكيف ان الوقت المستغرق في فك الثفرة اطول من التثفير وذلك بسب عملية الحساب لمعكوس المصفوفة. 
في هـــا الجـزء مـن البحـث سـوف نتطـرق إلـى عمليـة تنفيـذ البرنـامج الـذي تـم تتفيـذه باسـتخدام لغة6]20isual Basic 6.0][2] ويمكن تتبع خطوات التنفيذ عن طريق المراحل الآتية:

7. - 2. - الجزء التنفيذي للبرنامج

عند تنفيذ البرنامج سوف تظهر الشاشة الآتية كما في الشكل (7) والتي تحوي علجزئين: الاول: الجزء الاعلى خاص بالتشفير مع حساب الوقت المستغرق للتشفير • الثاني: الجزء الاسفل خاص بفك الثفرة مع حساب الوقت المستغرق لفك الثفرة.

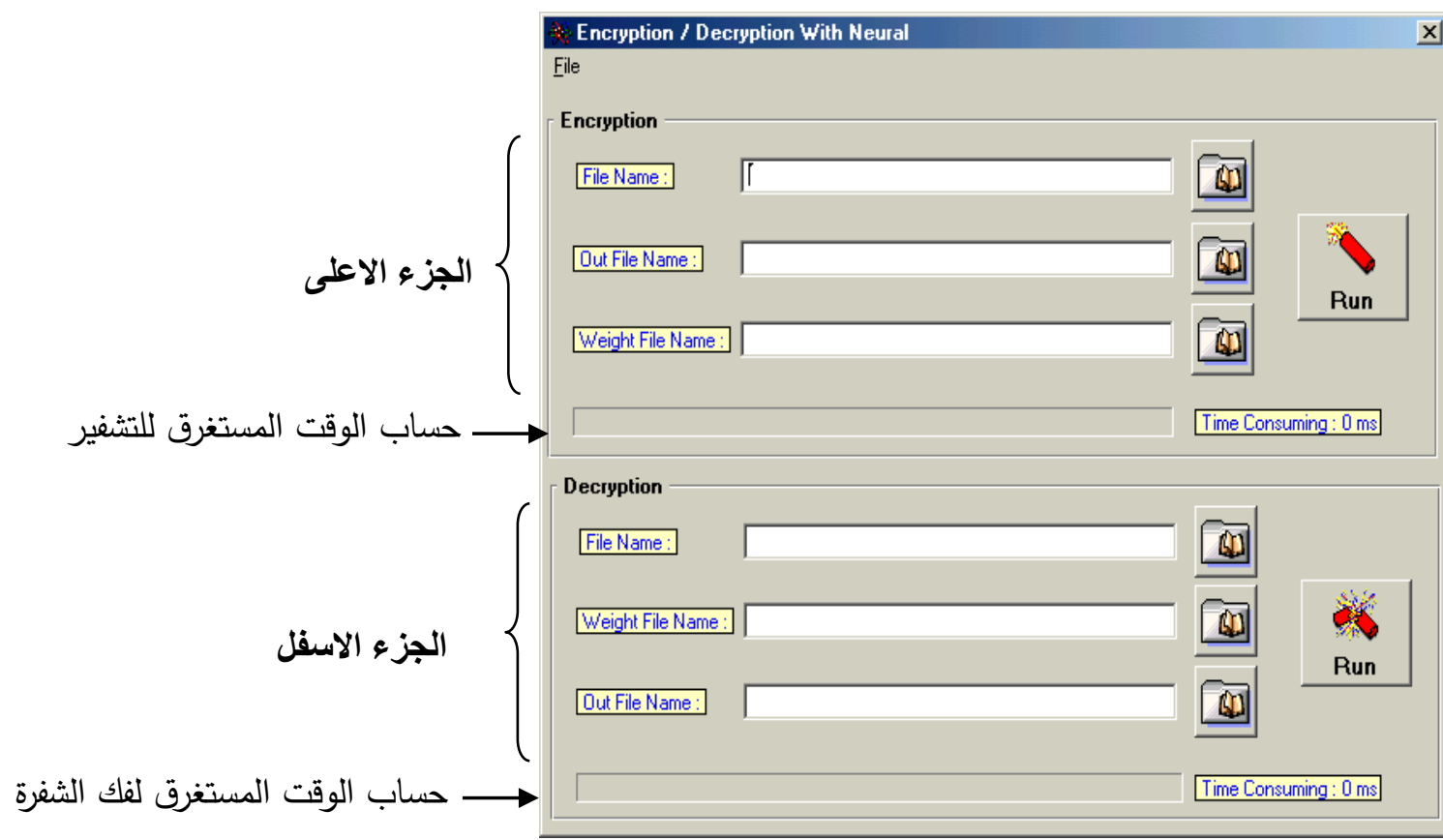

الثكل (7) نافذة بداية تنفيذ البرنامج

اذا كان النص ذات حجم صغير جداً فأن الوقت المستغرق في عملية التشفير وفك الثفرة يقاس بأجزاء الثانية لذلك فان الوقت المستغرق في التشفير وفك الثفرة يساوي اقل من الثانية الواحدة.

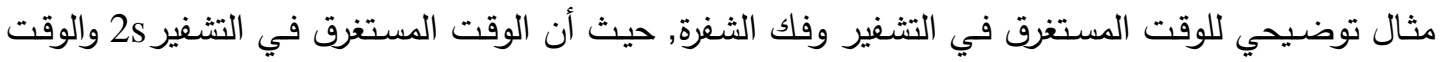
المستغرق في فك الثفرة 10s , نلاحظ بان الوقت في فك الثفرة اطول من وقت التشفير كما في الثكل(8). 


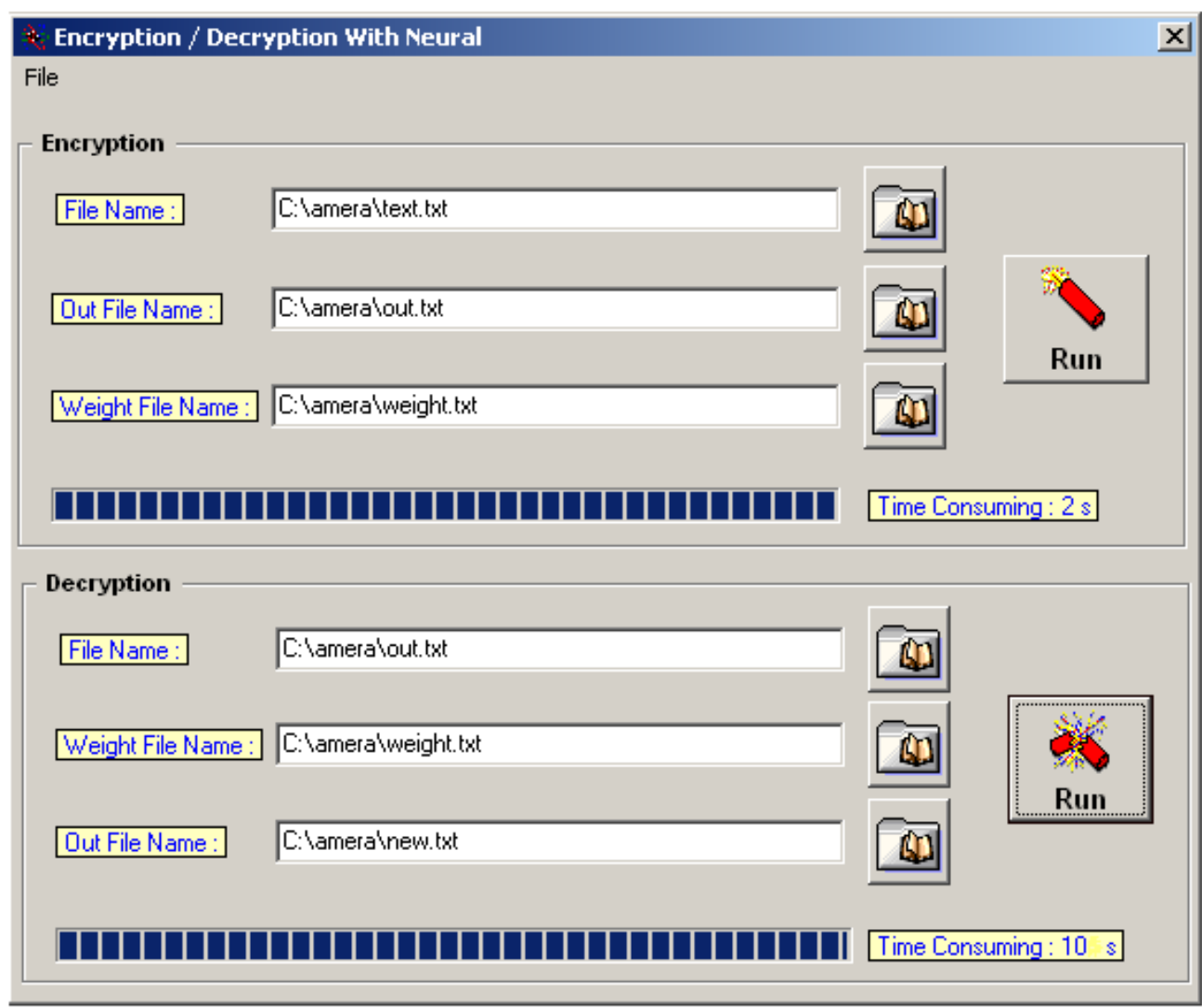

(8) الثكل (1) (1)

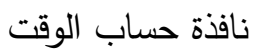

8

: مثال

• محتويات الملف (Test.txt) قبل عملية التشفير :

Microsoft Corporation

محتويات الملف (Output.txt) بعد عملية التثفير والتي تمثل القيم المقابلة لكل حرف:

\begin{tabular}{|r|r|r|r|r|r|r|}
\hline 229472 & 270831 & 350196 & 224732 & 316244 & 366492 & 232854 \\
\hline 277980 & 229456 & 295496 & 171597 & 280570 & 277579 & 276161 \\
\hline 304861 & 294848 & 317306 & 298337 & 347712 & 332726 & 282559 \\
\hline
\end{tabular}

محتويات الملف (New.txt) بعد عملية فك الثفرة:

Microsoft Corporation 


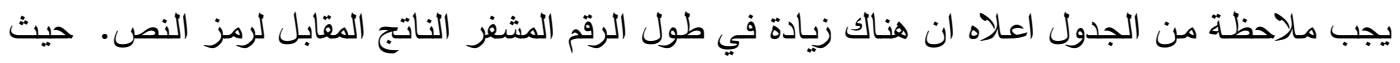
يحتاج الى تحليل مفصل لغرض ايجاد اقل نسبة من طول النص المشفر الى غير المشفر علماً ان العملية تتم

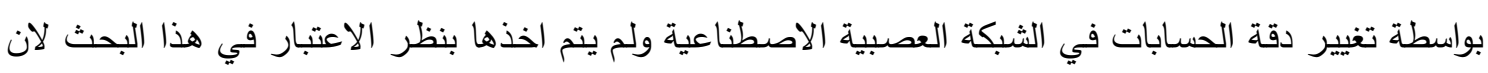
التركيز في امكانية عمل الشبكات العصبية الاصطناعية في التشفير وخاصة شبكة (Hebbian).

الاستنتاجات

إن عملية التشفير باستخدام الثبكة العصبية) Hebbian) تتضمن نوعاً من السرية العالية بسبب كبر المفتاح نسبياً وذلك لكونه يمثل مصفوفة ثنائية ذات أبعاد بحجم النص. وان عملية فلك الثفرة تتطلب وجود (مصفوفة الوزن) التي تمثل مفتاح (فك الثفرة). بالإضافة إلى أن حجم الملف الناتج من عملية التشفير يكون

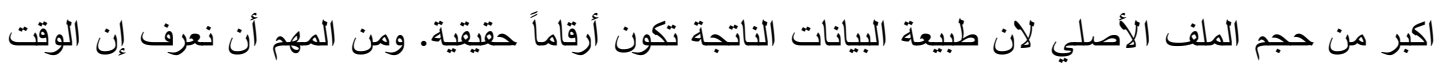

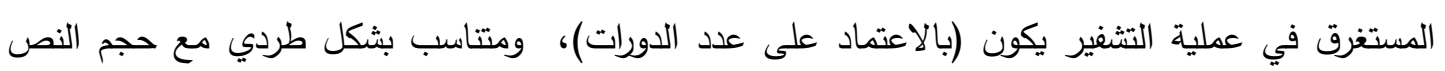
بالإضافة إلى أن الوقت المستغرق في عملية فلك الثفرة يكون كبيرا وذلك بسبب عمليب علية الحساب لمعكوس المصفوفة. ولقد تم التأكد من صحة النتائج في عملية فلك الثفرة بنسبة (100\%).

التوصيات:

1. التأكد المفصل من ان النظام الثفري أمين من خلال تطبيق الاختبارات الخاصة بالعشوائية على النص المشفر.

2. استخدام شبكة تتكون أكثر من طبقة للمقارنة بين الوقت المستغرق في عملية التشفير وشبكة (Hebbian)

أحادية الطبقة.

3. استخدام فكرة التشفير الكتلي في عملية تغذية مداخل الشبكة في حالة كون الملف المشفر كبيراً.

4. القيام على توفير سرية عالية على (مصفوفة الوزن) الناتجة من عملية التشفير (مصفوفة المفاتيح).

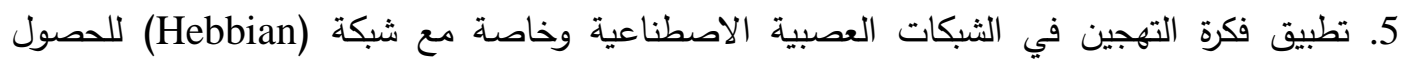
للحصول على وزن قياسي أمثل. وممكن أن نستغني عن معادلة الوزن في شبكة(Hebbian) باستخدام الخوارزمية الجينية.

6. إدخال علية كبس لمصفوفة الوزن باستخدام أسلوب الكبس بالثبكات العصبية (شبكة (Backprobagation لغرض تقليص حجم المصفوفة. 


$$
\begin{aligned}
& \text { المصادر } \\
& \text { العبيدي، محمود خليل ابراهيم (2000): "الثبكات العصبية الاصطناعية"، مجلة أبحاث الحاسوب، } \\
& \text { مدرس علم الحاسوب، الجامعة التكنلوجية، بغداد. } \\
& \text { الناظر، سائد محمود (1997): "كتاب المبرمح } 5.0
\end{aligned}
$$

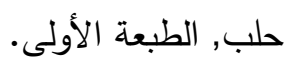

$$
\begin{aligned}
& \text { بدران, عامرة استقلال (2003):"استخدام شبكة (Hebbian) في التشفير"بحث ماجستير , كلية علوم } \\
& \text { الحاسبات والرياضيات, جامعة الموصل. } \\
& \text { سليمان، أنعام محمد (2002): "التداخل الشبكي الجيني (GA-Hf) لحل المسائل من نوع } \\
& \text { Np-problem (TSMP) } \\
& \text { هالفرسون، مايكل (1999): " Visual basic 6.0 خطوة خطوة"، الدار العربية للعلوم، لبنان. }
\end{aligned}
$$

